\title{
Recent Developments of Reflectarray Antennas for Reconfigurable Beams Using Surface-Mounted RF-MEMS
}

\author{
Eduardo Carrasco, ${ }^{1}$ Mariano Barba, ${ }^{1}$ Manuel Arrebola, ${ }^{2}$ and Jose A. Encinar ${ }^{1}$ \\ ${ }^{1}$ Departamento de Electromagnetismo y Teoria de Circuitos, Universidad Politécnica de Madrid, \\ Ciudad Universitaria s/n, 28040 Madrid, Spain \\ ${ }^{2}$ Area de Teoria de la Señal y Comunicaciones, Universidad de Oviedo, Edificio Polivalente de Viesques, Modulo 8, \\ Planta 1, 33203 Gijón, Spain \\ Correspondence should be addressed to Eduardo Carrasco, e.carrasco@ieee.org
}

Received 3 March 2012; Revised 18 June 2012; Accepted 23 June 2012

Academic Editor: Sandra Costanzo

Copyright (c) 2012 Eduardo Carrasco et al. This is an open access article distributed under the Creative Commons Attribution License, which permits unrestricted use, distribution, and reproduction in any medium, provided the original work is properly cited.

\begin{abstract}
Some of the most recent developments in reconfigurable reflectarrays using surface-mounted RF-MEMS, which have been developed at the Universidad Politécnica de Madrid, are summarized in this paper. The results include reconfigurable elements based on patches aperture-coupled to delay lines in two configurations: single elements and gathered elements which form subarrays with common phase control. The former include traditional aperture-coupled elements and a novel wideband reflectarray element which has been designed using two stacked patches. The latter are proposed as a low cost solution for reducing the number of electronic control devices as well as the manufacturing complexity of large reflectarrays. The main advantages and drawbacks of the grouping are evaluated in both pencil and shaped-beam antennas. In all the cases, the effects of the MEMS switches and their assembly circuitry are evaluated when they are used in a 2-bit phase shifter which can be extended to more bits, demonstrating that the proposed elements can be used efficiently in reconfigurable-beam reflectarrays.
\end{abstract}

\section{Introduction}

One of the main advantages of reflectarray antennas is the possibility of producing switching, scanning, or reconfiguration of the radiated beam if electronically controllable devices are included. The introduction of these elements allows the phase of the reflected field to be changed at each reflectarray cell. Several concepts have been developed to implement this kind of pattern flexibility, some of them provide a discrete phase control by using switches, such as pin diodes [1-3] or Micro-Electro-Mechanical Systems (MEMS) [4-8], and other realizations provide a continuous phase control by using varactor diodes $[1,9-11]$, ferroelectric materials [12], or liquid crystal [13,14]. Depending on the phase-control technique, the first step in the design of a reflectarray element to be used in steering or reconfigurable beam antennas is the suitable choice of the element configuration.
Reflectarray elements based on aperture-coupled patches using a microstrip delay line to control the phase of the reflected field [15], like that shown in Figure 1 have demonstrated a significant improvement in the bandwidth of large fixed-beam reflectarrays [16], through the introduction of true-time delay (TTD) which compensates the effects of the differential spatial phase delay [17]. These elements have also been used to produce shaped-beams, like those required by Local Multipoint Distribution System (LMDS) base stations [18]. The element consists of a square patch which is coupled to a variable length microstrip line using a rectangular slot. If the metallization dimensions are well adjusted, a phase response proportional to twice the length of the delay line can be achieved with very linear phase-delay within a range greater than $360^{\circ}$. This range is only limited by the room available on the cell to increase the microstrip line.

The aperture-coupled elements offer several advantages besides those related to the aforementioned TTD, such as 


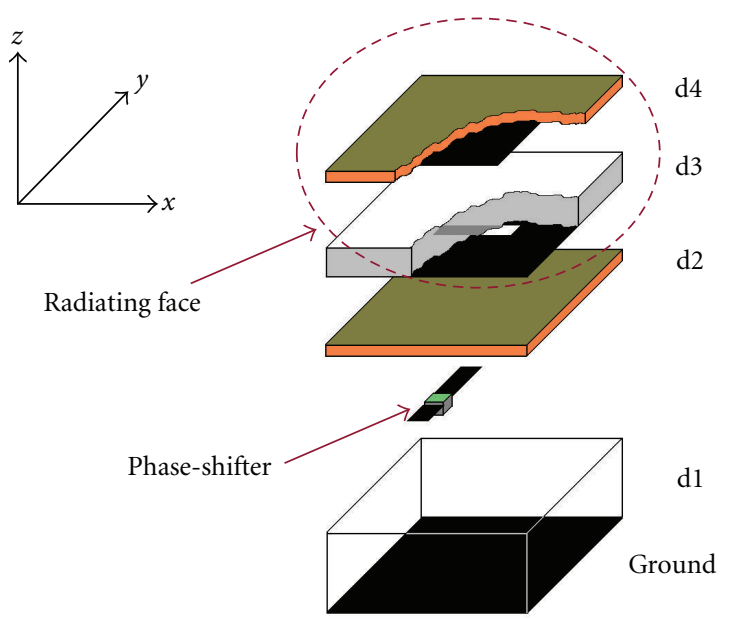

Figure 1: Expanded view of a single reconfigurable periodic element based on patches aperture-coupled to delay lines.

low losses, low cross polarization levels, and the possibility of implementing electronic devices which allow the phase to be controlled in reconfigurable antennas $[1,7,8,10,11]$. Two major benefits are the isolation of the radiating elements from the phase control network and phase-shifters because they are on opposite sides of the ground plane, as well as the possibility of gathering two adjacent elements to form a subarray with the aim of reducing both the number of control elements and manufacturing complexity [19], especially in large reflectarrays.

The proposed reflectarray element allows the phase of the reflected field to be controlled by modifying the electrical length of the phase delay. This can be done by dividing the microstrip into different segments which can be connected in series using electronic devices. MEMS switches offer attractive advantages because of their practically null power consumption, very high isolation, low insertion loss, total independence between DC and RF signals, and high level of integration because they can be monolithically implemented in the delay-line wafer $[4,7]$. However, this monolithically integration is not economically affordable in X-band antennas requiring the use of other alternatives for the manufacturing. One possibility is the use of surface-mounted MEMS which can be connected to the microstrip lines by means of a standard wire bonding process [8].

In the following sections the aperture-coupled reflectarray element is shown as a well-situated solution to implementing reconfigurable-beam antennas using surfacemounted MEMS. Three practical cases are presented at $10.40 \mathrm{GHz}$ : single elements, gathered elements, and a wideband single element obtained by stacking two patches.

\section{Reconfigurable Element Based on Surface-Mounted Devices}

2.1. Reconfigurable Single Element Based on Patches, ApertureCoupled to Delay Lines. For the present work, MEMS series switches designed and manufactured by CEA-LETI Minatec
[20] have been used. These MEMS switches are implemented in a $50 \Omega$ coplanar waveguide (CPW) with a gap in the signal conductor and a silicon nitride fixed-fixed membrane with a patterned metallic contact in the centre [21, 22]. This contact gives continuity to the signal line of the CPW when a DC voltage is applied between two symmetric electrodes and the ground plane. The devices have been supplied as individual dice after the wafer cutting, with side dimensions of around $800 \mu \mathrm{m}$ and $80 \mu \mathrm{m}$ for the pad width. These dimensions combined with the fact that the dielectric substrate which will contain the MEMS is very thin make the use of long gold wires necessary for the bonding which introduces inductive effects and an inherent phase-shift which must be taken into account.

The proposed electronically controllable element can be analysed using a cascading technique, as shown in Figure 2. With a full wave approach using CST Microwave Studio [23], the two-port scattering matrix of the fixed phase reflectarray element is obtained under local periodicity conditions. In this case, the first port refers to the top surface of the upper dielectric, while the second port refers to the end of a microstrip line. The reflection coefficient in the free space of the reconfigurable element is obtained by loading the first port with the intrinsic impedance of air and the second port with the impedance which represents the MEMS assembly plus a microstrip segment which can be open ended or connected to another MEMS assembly. The MEMS assembly which includes the MEMS device and the wire bonding lines is obtained through an equivalent circuit, which has been deduced and demonstrated in [8]. Depending on the voltage applied to the switch, the MEMS contact can be modelled as a series resistance or a capacitance. For the particular case of the MEMS used in this work, these values are, respectively, $R_{s}=2.15 \Omega$ and $C_{\mathrm{up}}=1 \mathrm{fF}$.

Prior to the reconfigurable element analysis, the design of the passive element must be carried out. The proposed single element has been designed using a local periodicity approach with a period of $18.5 \mathrm{~mm} \times 18.5 \mathrm{~mm}$, which means $0.64 \lambda$ at $10.40 \mathrm{GHz}$. The period has been chosen as a tradeoff in order to allow more room for long delay lines, for the control devices and their associated DC lines, on one side, and to avoid the appearance of grating lobes, on the other side. For a whole reflectarray, the grating lobes will appear for incidence angles greater than $34^{\circ}$. The square patches are $9.1 \times 9.1 \mathrm{~mm}^{2}$, the slot $7.0 \times 1.4 \mathrm{~mm}^{2}$, and the delay line width $0.388 \mathrm{~mm}(70 \Omega)$. This impedance has been chosen with the aim of improving the matching between the microstrip line and the MEMS assembly, taking into account that the $25 \mu \mathrm{m}$ of the gold wires present high impedance, in the order of $200 \Omega$. With the aim of obtaining a very linear phase curve of the reflection coefficient as a function of the length of the line, the matching stub of the element has been fixed to $1.90 \mathrm{~mm}$, measured from the centre of the slot. The main features of the dielectric substrates are shown in Table 1.

Figure 3 shows the reflection coefficient in free space for the passive element as a function of the line length. The losses of the element are around $0.25 \mathrm{~dB}$ at the central frequency. A practically linear phase delay is obtained in a bandwidth of more than $10 \%$, with a phase range of at least 2 cycles of $360^{\circ}$. 


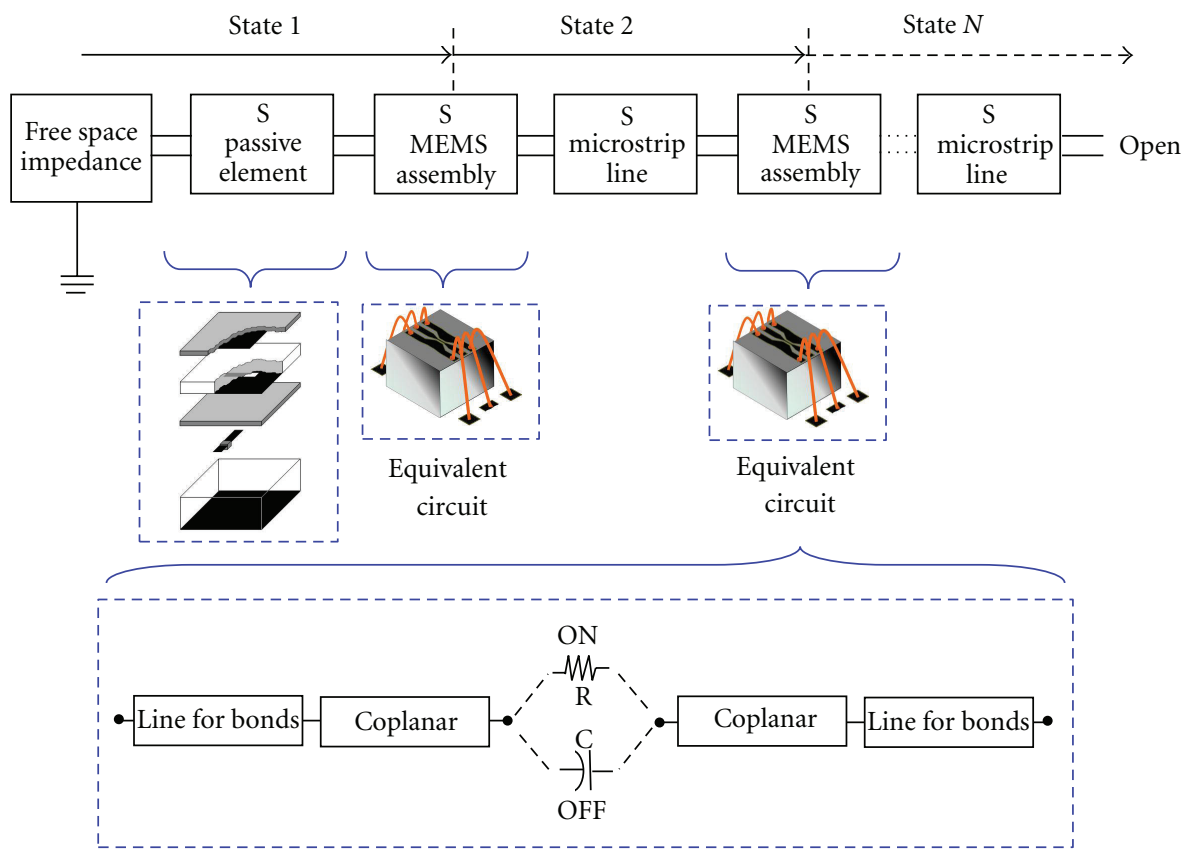

Figure 2: Modelling of the reflectarray element with phase control.

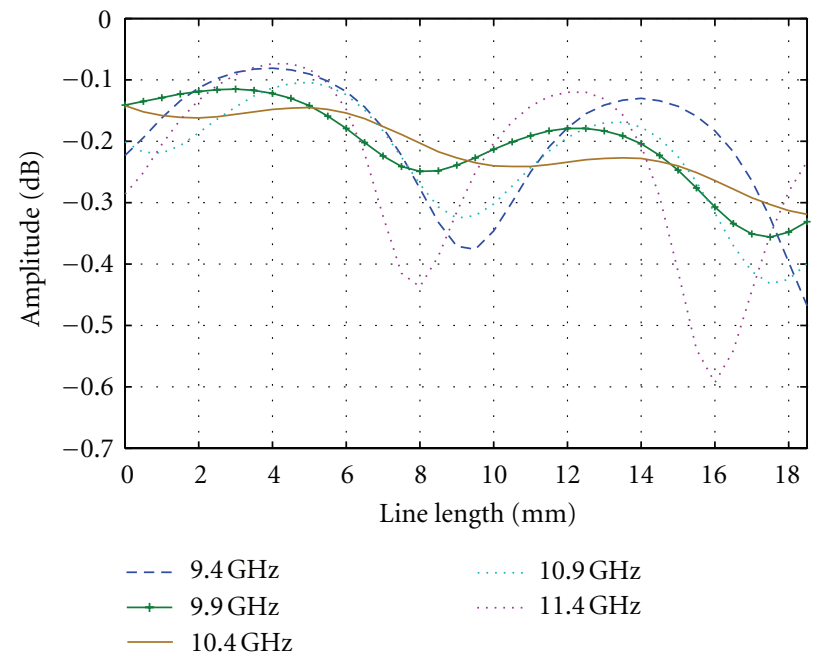

(a)

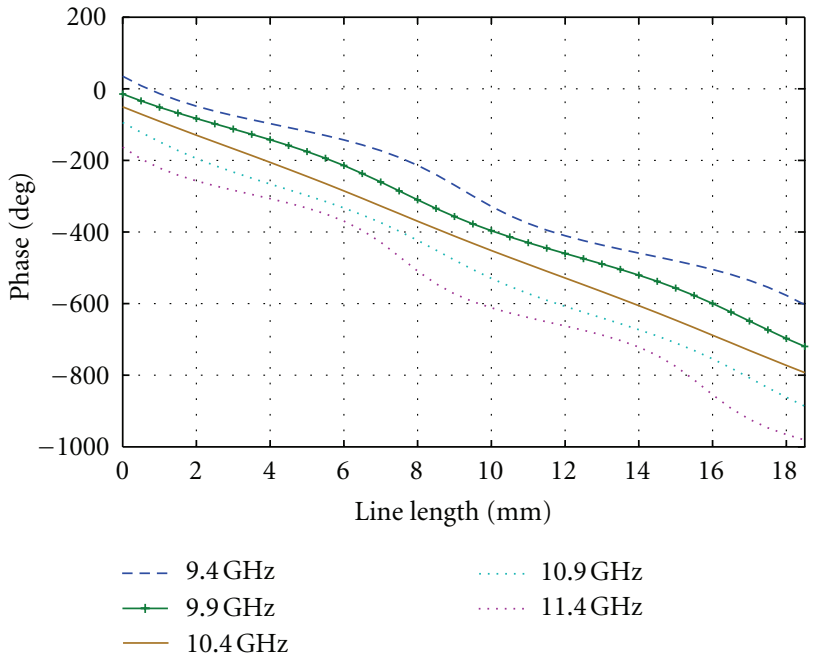

(b)

Figure 3: Reflection coefficient in free space for the passive single element as a function of the delay line length. (a) Amplitude. (b) Phase.

TABLE 1: Dielectric substrates.

\begin{tabular}{lccc}
\hline Layer & $\varepsilon_{r}$ & $\tan \delta$ & Thickness $(\mathrm{mm})$ \\
\hline $\mathrm{d} 4$ & 3.380 & 0.0035 & 0.508 \\
$\mathrm{~d} 3$ & 1.067 & 0.0002 & 2.000 \\
$\mathrm{~d} 2$ & 3.380 & 0.0035 & 0.305 \\
$\mathrm{~d} 1$ & 1.000 & 0.0002 & 7.200 \\
\hline
\end{tabular}

This phase range is only limited by the cell room and can be easily incremented by bending the delay line to form an $\mathrm{L}$ or a $U$ shape as in [15]. Once the passive element has been designed, the effects of connecting the MEMS devices can be evaluated. Here, it is important to note that, depending on the application, the proposed element can be tuned to any phase value (switching beam) or to certain discrete values (reconfigurable beam). Here, the element has been evaluated as a 2-bit phase shifter which allows 4 states to be produced equally separated by $90^{\circ}$, but can be extended to more states by increasing the number of switches which are connected to the delay line. The amplitude of the reflection coefficient for the 4 states as well as for the passive element is shown 


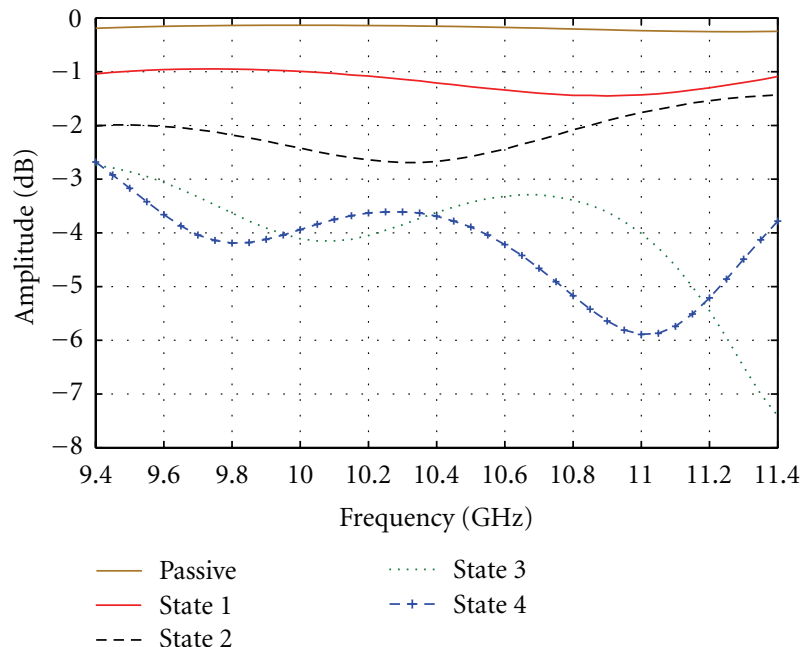

(a)
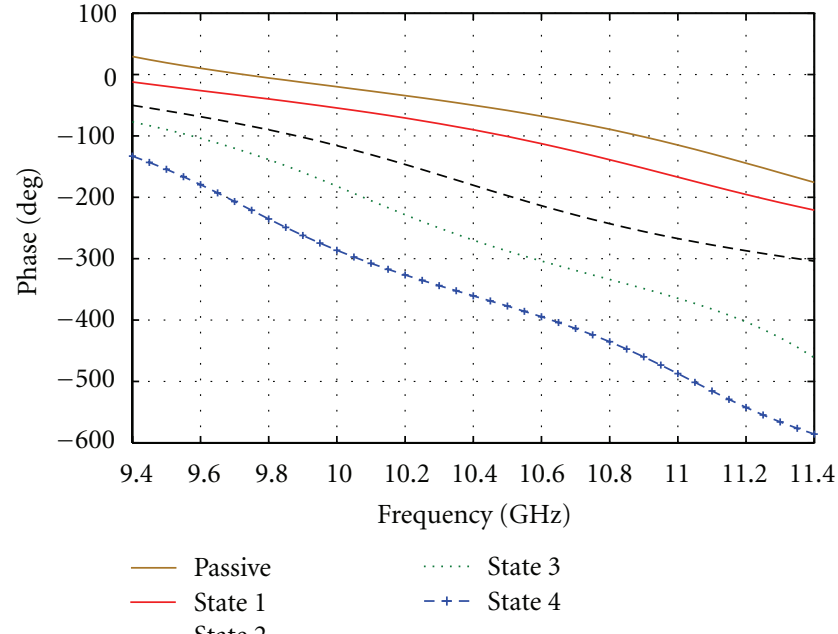

(b)

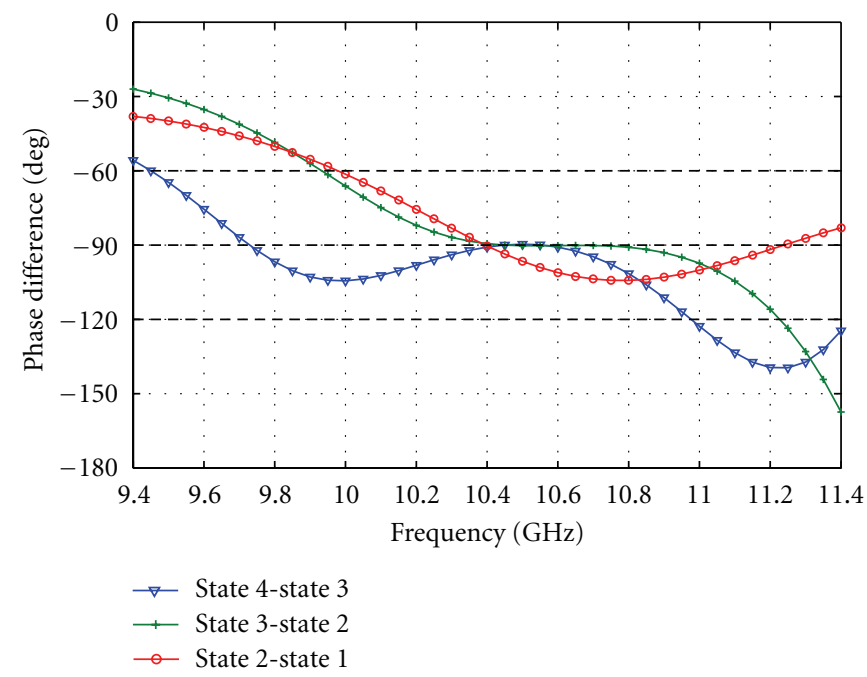

(c)

Figure 4: Reflection coefficient versus frequency for a 2-bit reflectarray element in a periodic environment. (a) Amplitude. (b) Phase. (c) Phase difference between the adjacent states.
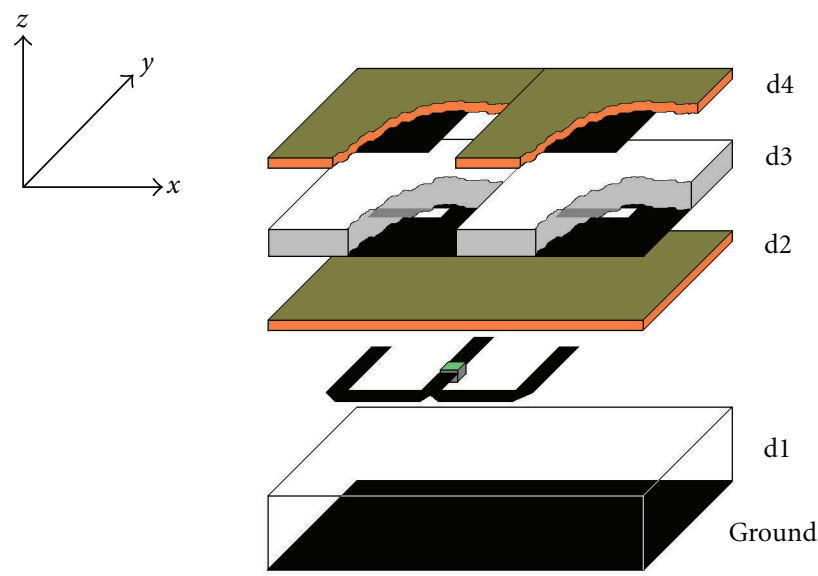

FIGURE 5: Expanded view of a gathered reconfigurable element based on patches, aperture-coupled to delay lines. 


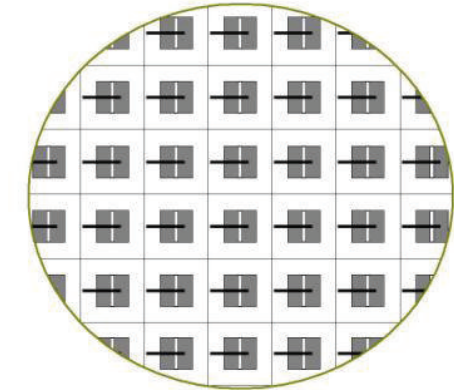

(a)

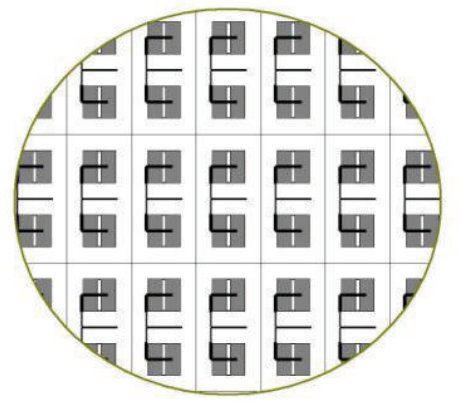

(c)

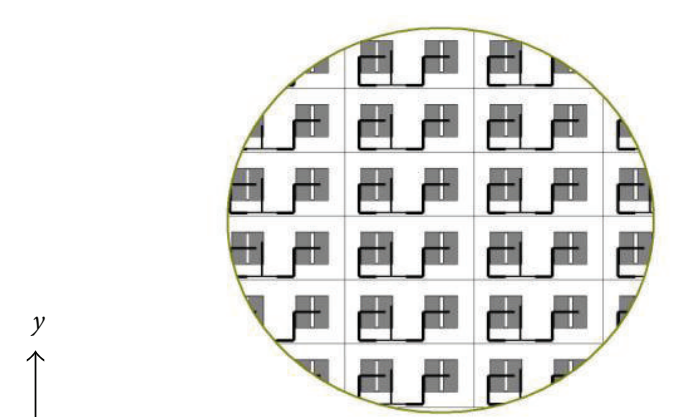

(b)

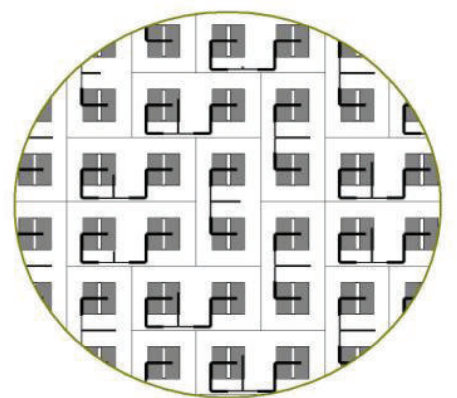

(d)

Figure 6: Detailed view of different lattices for a reflectarray based on aperture-coupled elements. (a) Single elements. (b) Gathered elements along the $x$-axis. (c) Gathered elements along the $y$-axis. (d) Irregular gathering.

in Figure 4(a), in the frequency band from $9.4 \mathrm{GHz}$ to $11.40 \mathrm{GHz}$ which is the band in which the MEMS assembly has been previously characterized and validated [8]. The losses of the element are mainly produced by the $Z_{s}$ which is presented between the contact in the membrane and the CPW when the switch is in the ON position and by the mismatch produced between the microstrip and the wire bonding which has more impact as the bonding wires are longer. These large wires are also responsible for the inherent phase-delay introduced by the MEMS assembly, which is around $60^{\circ}$ when the MEMS is in the OFF position and $120^{\circ}$ when the MEMS is ON. Phase values lower than these can be achieved by introducing an extra $360^{\circ}$ cycle. The phase response of the element is shown in Figure 4(b). If the phase variation between adjacent states is limited to $+30^{\circ}$, which is a very acceptable value for a 2-bit phase-shifter, the proposed reflectarray element provides a $10 \%$ bandwidth, as can be seen in Figure 4(c).

2.2. Reconfigurable Gathered Elements Based on Patches, Aperture-Coupled to Delay Lines. Large reflectarrays with reconfigurable beams can require hundreds or thousands of electronic devices with their associated DC lines to control the phase of the reflected field at each element. This means a significant increase in the cost of the antenna as well as the manufacturing complexity. The number of control devices can be drastically reduced if sparse elements are used [24]. However the reflection produced by the ground plane in the zones between the elements makes the design of the reflectarray antenna difficult. Another alternative is the grouping together of two or more neighbouring elements to form a subarray $[19,25]$. Because of the independence between the radiating face and the phase-shifting device, the proposed aperture-coupled element allows two or more elements to be controlled with the same delay line without a significant degradation in the reflectarray performance. Figure 5 shows the expanded view of a gathered reflectarray element where two aperture-coupled patches are controlled by the same delay line. It should be noted that, for the design of the gathered element, first a single-element must be designed in order to obtain a reflection coefficient with low losses and a very linear phase response, proportional to twice the length of the delay line, as in Section 2.1. The second step is to design a microstrip circuit (T-junction, quarter of wavelength transformers, bends and delay line) in order to joint two adjacent elements. The two elements are gathered using the previously designed microstrip circuit and a small readjustment of the individual elements must be performed in order to compensate the effects introduced by the grouping and therefore to keep the linear phase response.

As expected, the gathering of the elements in pairs will change the response of the reflected field by two factors. Firstly, the phase required to produce the desired beam, which depends on the radiation pattern, the feed and elements position, is limited in resolution. This means that the phase delay that must be provided by the two adjacent elements, controlled by the same delay line, must be adjusted to the value that introduces the lower phase error (quantization error) with respect to the original phase which was synthesized for the two single elements. An increase in the grating lobes level will also be produced because the period of the sub-array is greater than the wavelength. This second factor can have more impact on the antenna performance. 


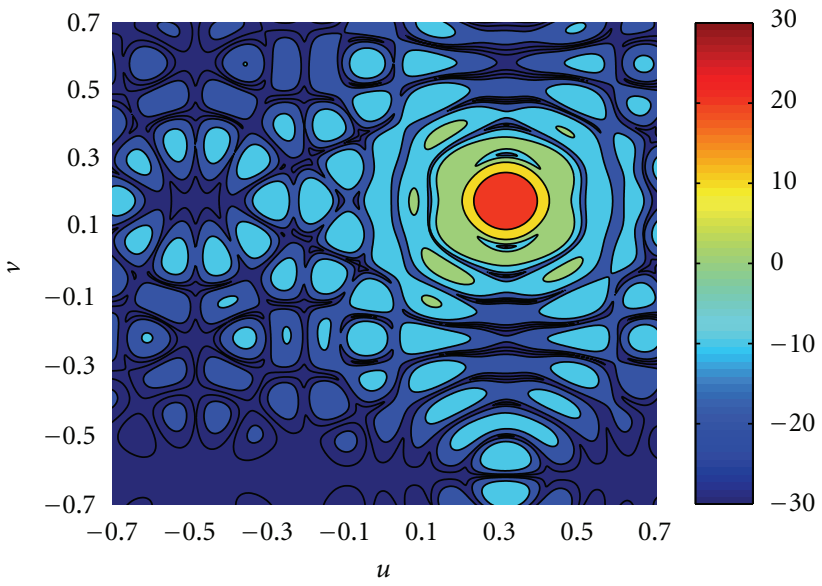

(a)

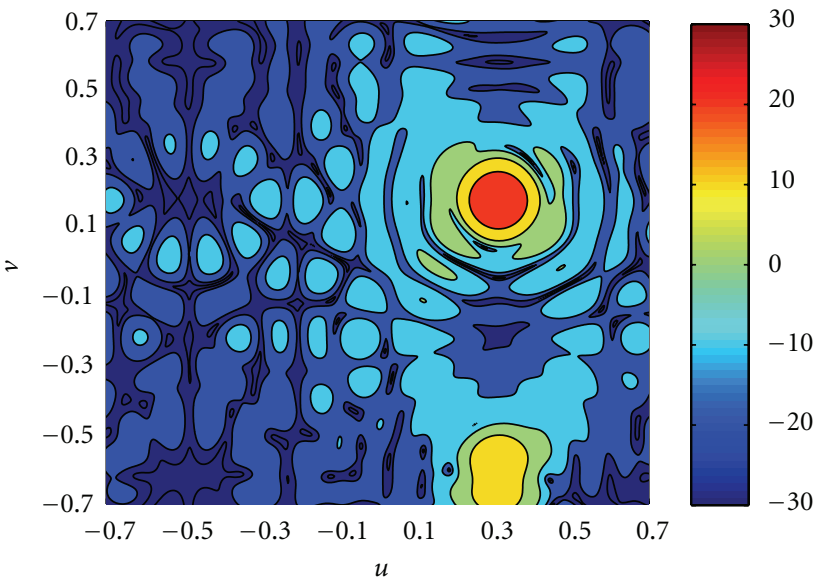

(c)

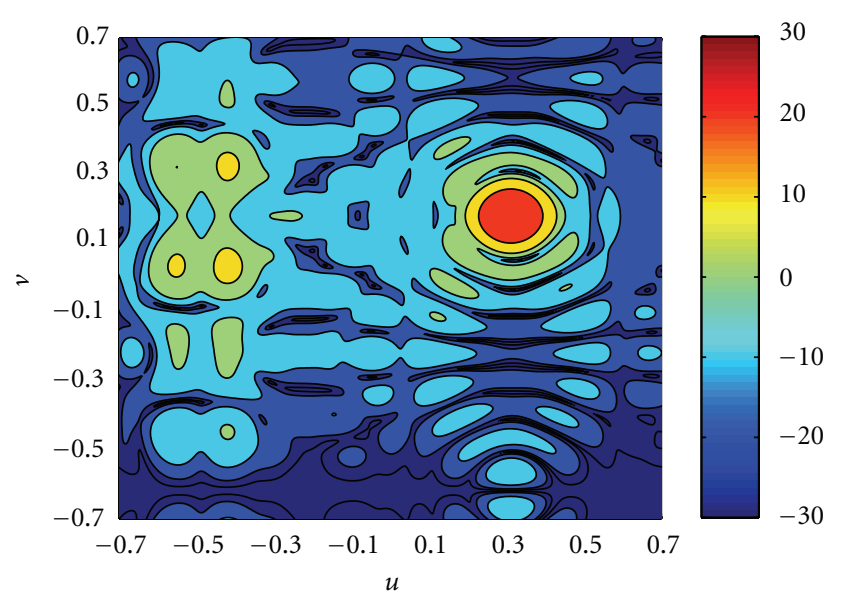

(b)

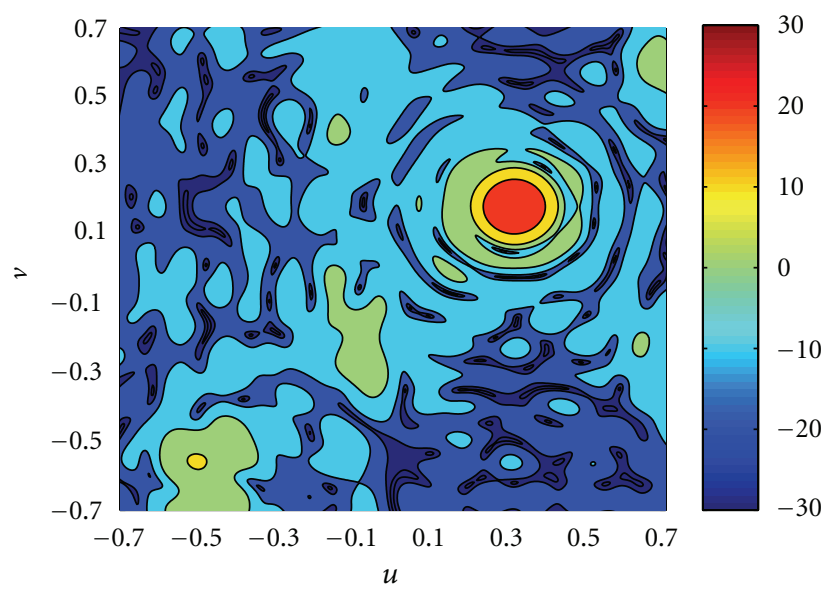

(d)

FIGURE 7: Radiation patterns produced with the pencil-beam reflectarray antenna using different lattices. (a) Without any grouping. (b) Grouping along the $x$-axis. (c) Grouping along the $y$-axis. (d) Grouping in an irregular lattice.

The undesired grating lobes can be drastically reduced or even eliminated if the gathering is carried out using irregular lattices $[26,27]$. The effects of the grouping have been evaluated for both cases: pencil- and shaped-beam reflectarrays with the features shown in Table 2, using ideal elements. In the two cases different lattices for the gathering have been analysed as can be seen in Figure 6. The first lattice corresponds to the original antenna, where the elements have not been grouped together conserving all the required phase values and the original cell period. The second lattice corresponds to a grouping along the $x$-axis, while in the third lattice the grouping has been carried out along the $y$-axis. Finally, the fourth lattice called an irregular lattice corresponds to a nonregular combination of elements grouped together along both the $x$-axis and the $y$-axis breaking the period of the lattice along the main planes.

For the case of a pencil-beam reflectarray radiating in certain direction defined by the angles $(\theta, \varphi)$ in spherical coordinates, the impact of the grating lobes will be greater as $\theta$ increases. As an example, the case of a circular reflectarray antenna radiates a beam towards $11^{\circ}$ in a switching plane
TABLE 2: Reflectarray antennas features.

\begin{tabular}{lcc}
\hline & Pencil beam & Shaped beam \\
\hline Diameter $(\mathrm{mm})$ & 324 & 396 \\
Number of elements & $244(122$ subarrays $)$ & $362(181$ subarrays $)$ \\
Feed position $(\mathrm{mm})$ & & \\
$x$ & -100 & -140 \\
$y$ & 0 & 0 \\
$z$ & 330 & 450 \\
\hline
\end{tabular}

which is tilted at $18.3^{\circ}$ in respect the horizon. The radiation patterns associated to the corresponding phase distributions obtained with the aforementioned lattices have been computed in directivity, by dividing by the total power radiated by the feed, and including the spillover losses, that is without taking into account the losses of the elements since the objective here is to evaluate the impact of the gathering for each lattice. The feed horn has been modelled as a $\cos ^{q}$ function, being $q=10$, at the central frequency of $10.40 \mathrm{GHz}$. 


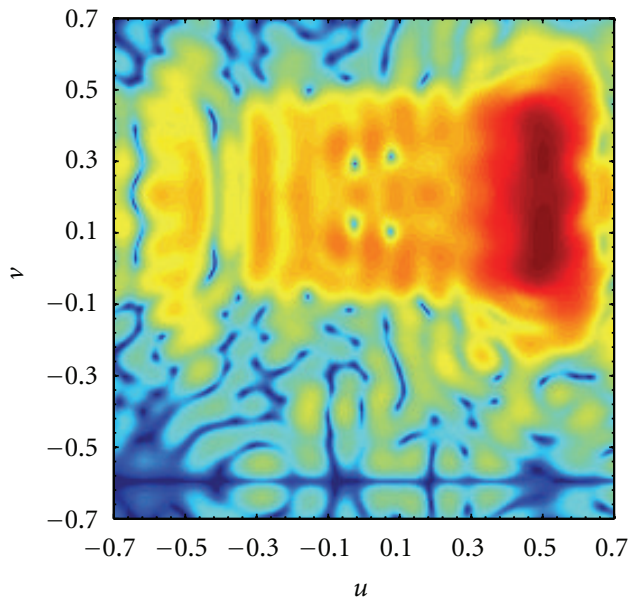

(a)

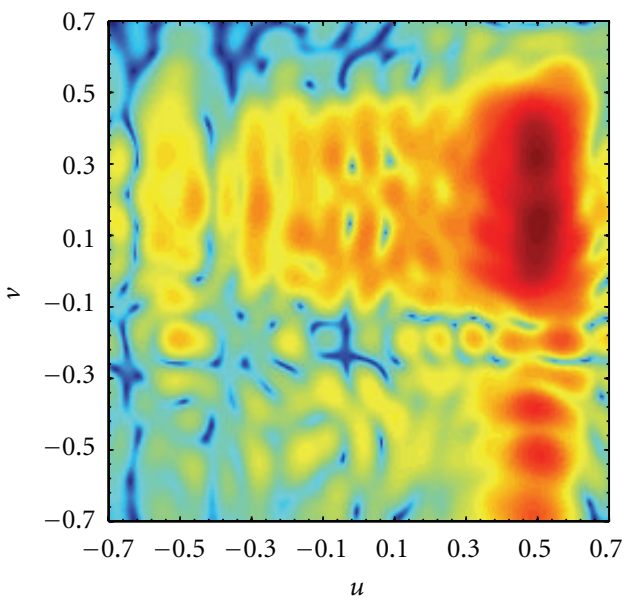

(c)
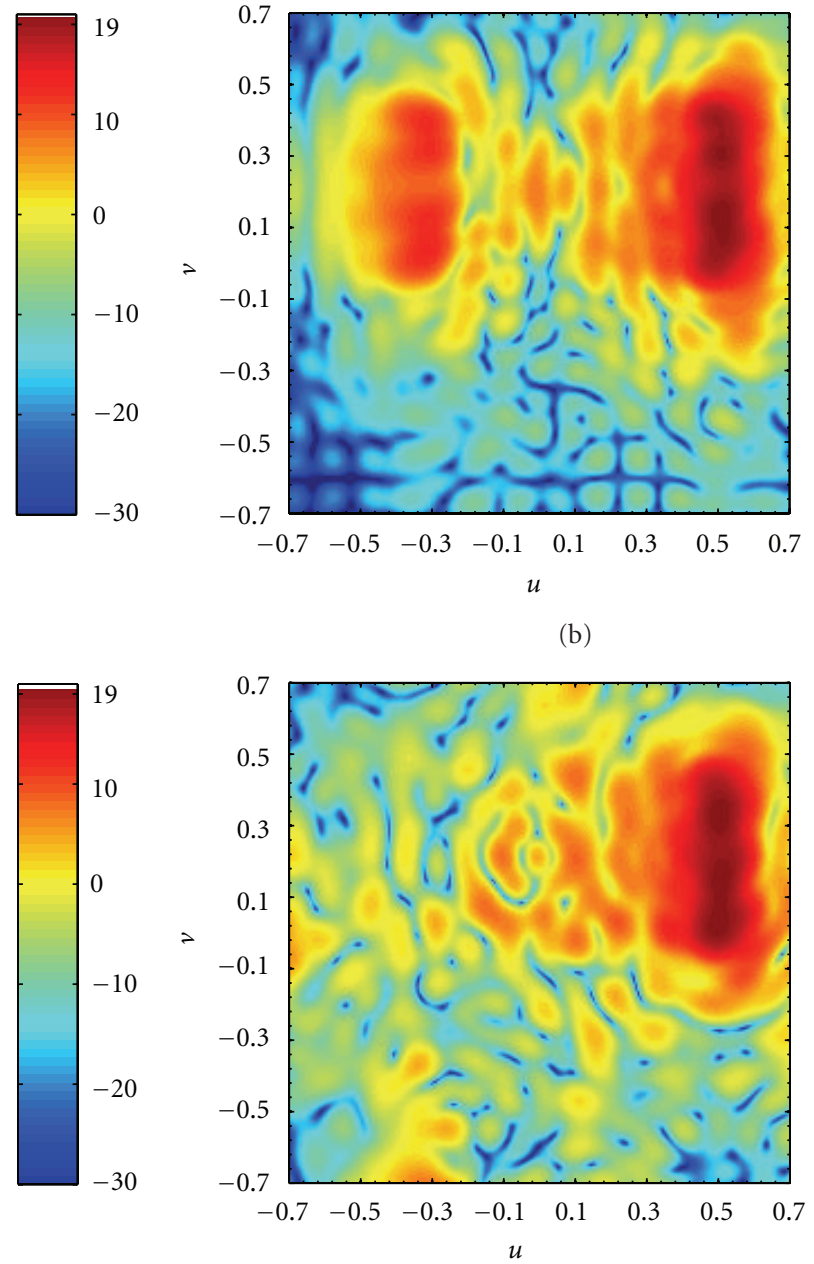

(d)

FIGURE 8: Radiation patterns produced with the shaped-beam reflectarray antenna using different lattices. (a) Without any grouping. (b) Grouping along the $x$-axis. (c) Grouping along the $y$-axis. (d) Grouping in an irregular lattice.

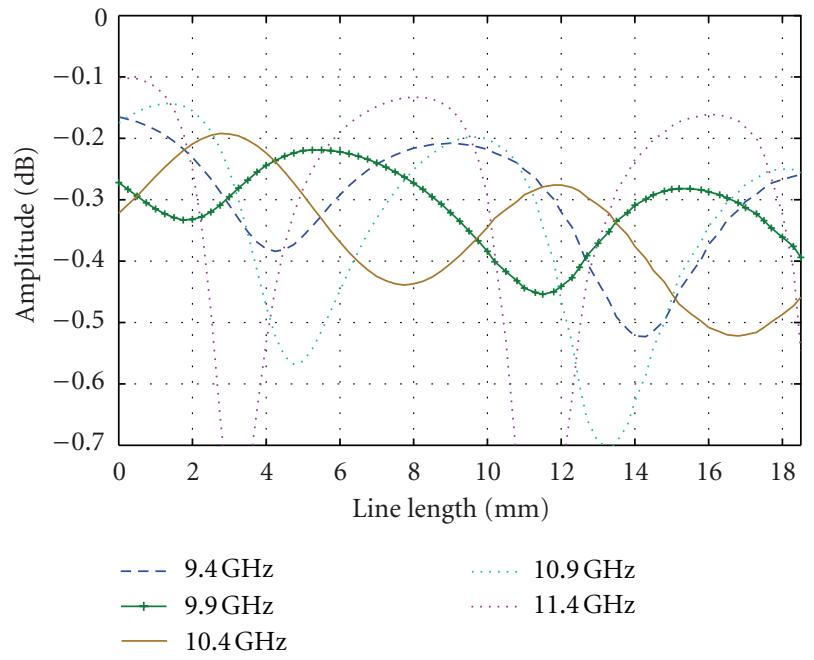

(a)

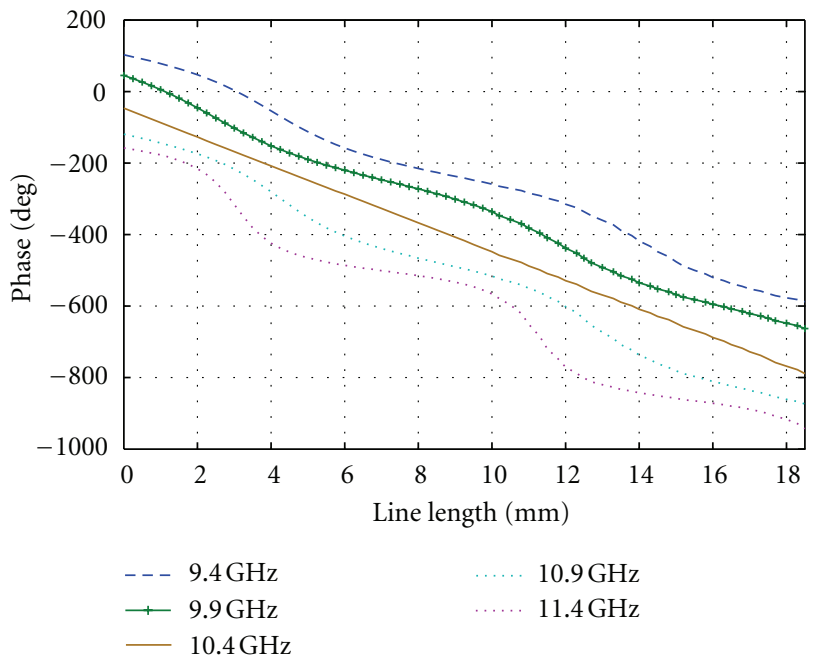

(b)

FIGURE 9: Reflection coefficient in free space for the two-element subarray. (a) Amplitude. (b) Phase. 


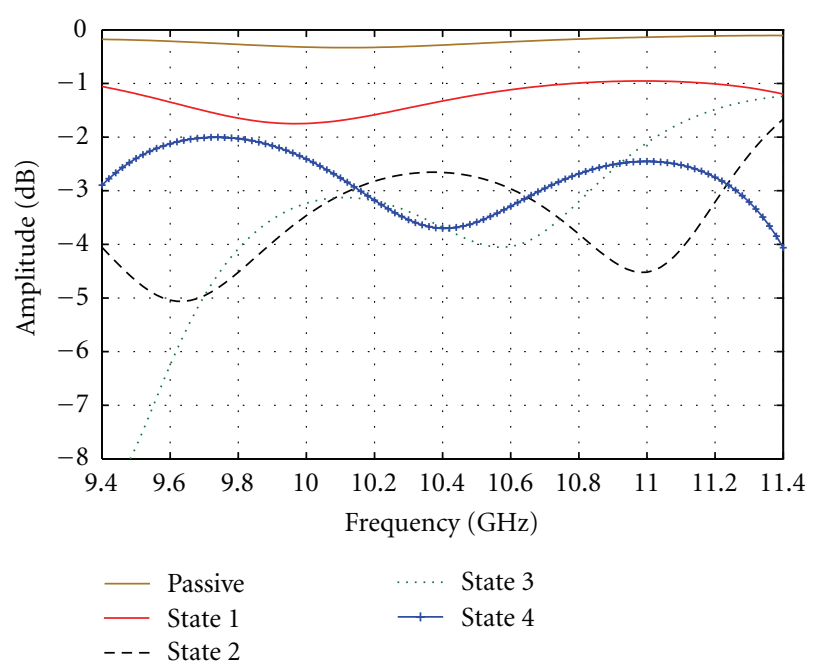

(a)

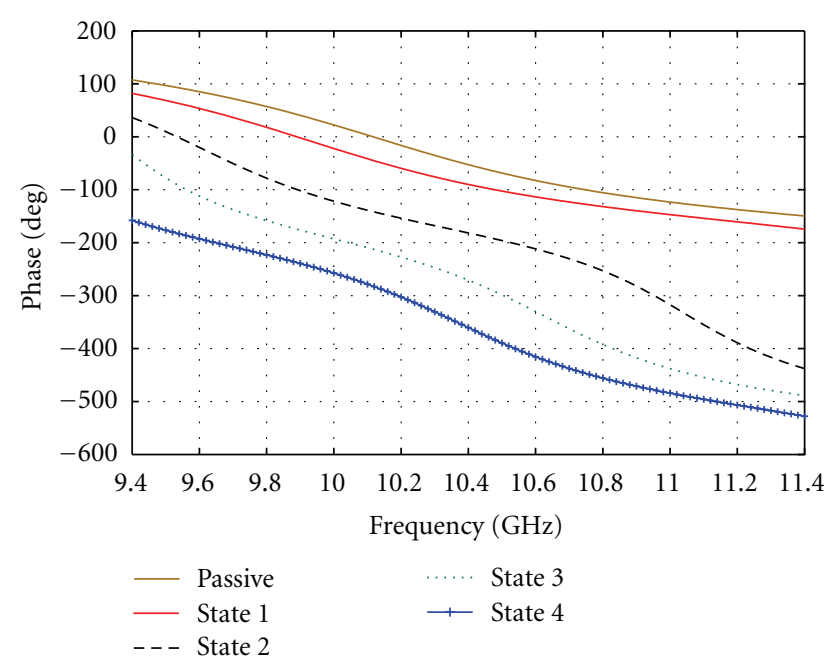

(b)

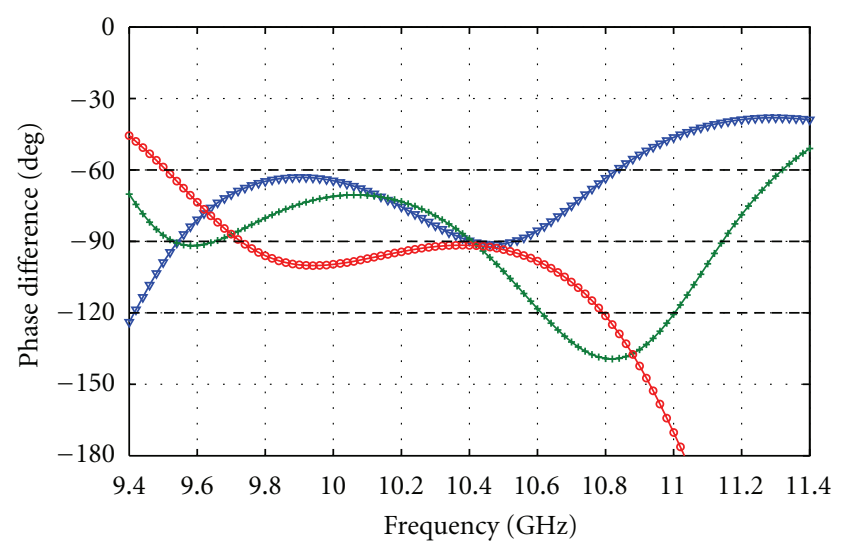

$\rightarrow$ State 4 -state 3

$\rightarrow$ State 3 -state 2

$\rightarrow$ State 2 -state 1

(c)

Figure 10: Reflection coefficient for the 2-bit gathered element in a periodic environment. (a) Amplitude. (b) Phase. (c) Phase difference between the adjacent states.

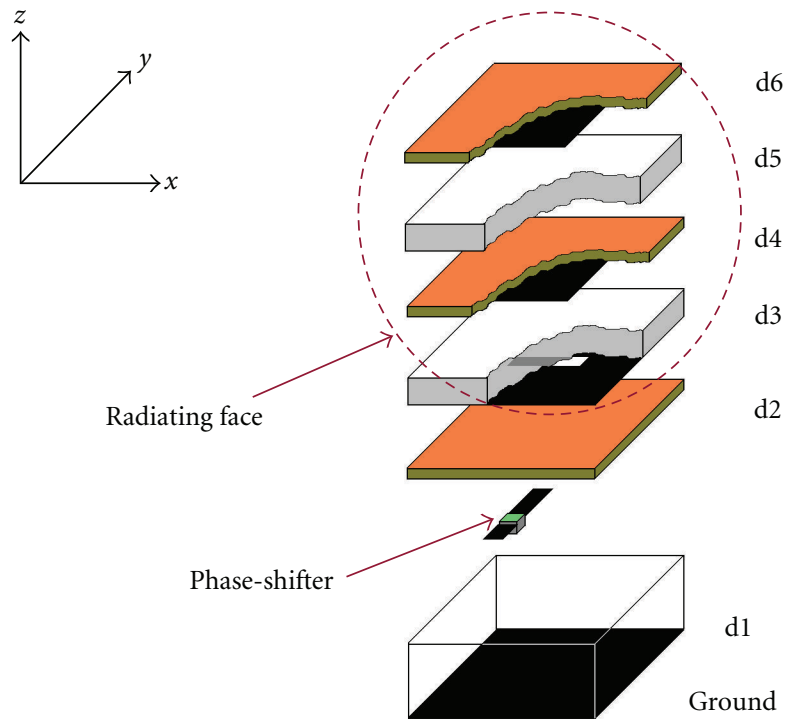

FIGURE 11: Expanded view of a single reconfigurable element based on patches, aperture-coupled to delay lines with two stacked patches.

Figure 7 shows the three-dimensional radiation patterns for the respective cases in the $u-v$ coordinates, where $u=$ $\sin \theta \cos \varphi$ and $v=\sin \theta \sin \varphi$. For the reference pattern, which is the pattern without any grouping and therefore compensating the phase at each individual element, there are no grating lobes and the directivity is $28.4 \mathrm{~dB}$. For the reflectarray with gathered elements in the $x$-direction, the grating lobes appear in the elevation plane, while in the case of gathered elements in the $y$-direction the grating lobes appear in the azimuth plane and can eventually cause interference. For these cases the directivity values are 27.71 and 27.18, respectively. Finally, in the non-regular lattice the grating lobes are produced in a region furthest from the main lobe with a very small width.

In the second example, a shaped-beam reflectarray has been designed to produce a square cosecant beam in the elevation plane tilted by $30^{\circ}$ and a $30^{\circ}$ sector beam deviated $15^{\circ}$ with respect to the antenna boresight. The radiation patterns for this antenna have also been obtained in directivity, using the different lattices for the grouping together of the elements. Figure 8 shows the 3 -D radiation patterns also in the $u-v$ coordinates. As expected, there are no grating lobes for the case without grouping. In the antenna with gathered elements along the $x$-axis, the grating lobes appear in the elevation plane with levels of only $5 \mathrm{~dB}$ below the main beam, which is an inadmissible value for the majority of the applications. For the antenna with gathered elements along the $y$-axis, a grating lobe appears in the azimuth plane with a level of $7 \mathrm{~dB}$ below the main lobe. As in the previous case, this lobe must be eliminated or drastically reduced. Finally, by using an irregular lattice which breaks the periodicity of the grouping, the grating lobe is practically eliminated. In all of the cases, the directivity values are greater than $16.25 \mathrm{~dB}$, 


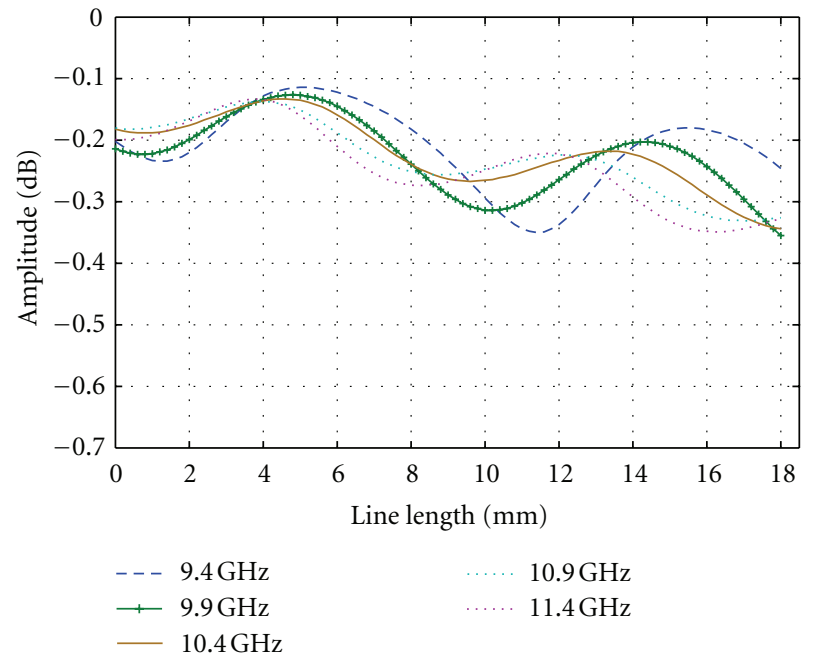

(a)

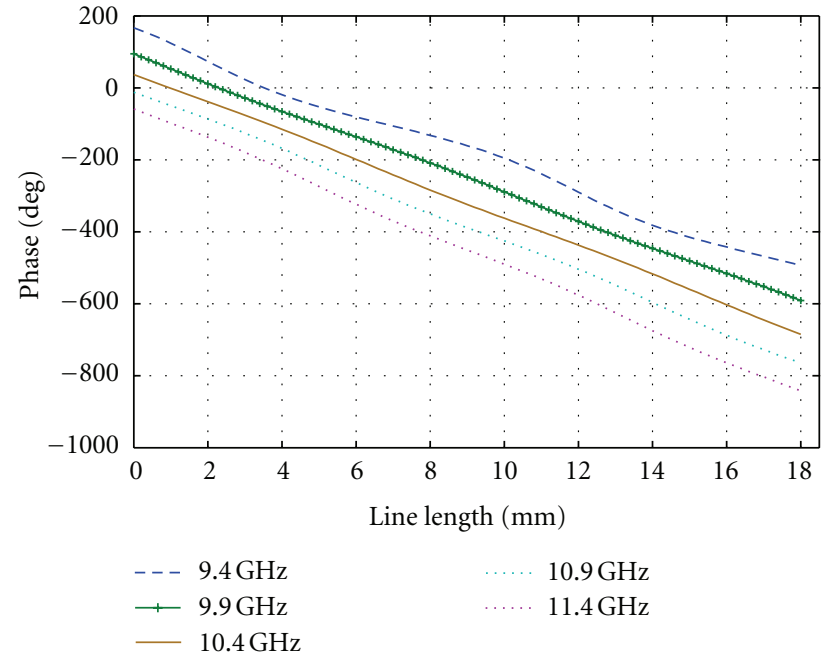

(b)

FIGURE 12: Reflection coefficient in free space versus the line length for the reflectarray element with two stacked patches. (a) Amplitude. (b) Phase.

fulfilling with the required specifications for this particular case.

Once the impact of grouping two adjacent elements together with a common control has been evaluated, a reconfigurable gathered element is presented. As in the case of a reconfigurable simple element, the first step is to design a passive element which will be connected to the phase control network. The modelling approach of Figure 2 is also valid if the scattering matrix of the gathered element is used in the place of the single element scattering matrix. The dielectric materials are the same as in the case of a single element, but the dimensions of the element have been updated as follows in order to keep the linearity of the phase response. The size of the sub-array is $36 \mathrm{~mm} \times 18 \mathrm{~mm}$, which means $1.24 \lambda \times 0.62 \lambda$, at the designing frequency of $10.40 \mathrm{GHz}$. As can be observed, the period of the single element $(18.5 \mathrm{~mm})$ has been slightly reduced $(18.0 \mathrm{~mm})$. The side of the square patches is $9.1 \mathrm{~mm}$ and the rectangular slots are $8.9 \mathrm{~mm} \times$ $1 \mathrm{~mm}$. In this case the width of the microstrip line is also 0.39 to obtain a $70 \Omega$ characteristic impedance. The matching stub length is $1.87 \mathrm{~mm}$, measured from the centre of the slot. Figure 9 shows the reflection coefficient in free space of the proposed sub-array as a function of the line length in the band from $9.40 \mathrm{GHz}$ to $11.40 \mathrm{GHz}$. The average losses at the central frequency of $10.40 \mathrm{GHz}$ are $0.35 \mathrm{~dB}$ which is slightly greater than that of a single element. At extreme frequencies the losses increase as a result of the gathering. The frequency curves remain very linear from $9.4 \mathrm{GHz}$ to $10.40 \mathrm{GHz}$, with an increase in the slope at the higher frequencies which was expected as a result of the effects introduced by the T-junction and the impedance transformers required in the element grouping.

Note that the reflection coefficient for each sub-array formed by two gathered elements has been computed using a local-periodicity approach (Floquet's conditions). This approach gives very accurate results in this kind of element because independently on the lattice (along $x$, along $y$ or irregular), all the elements are exactly equal in the patches and slots layers. The only difference between neighbouring elements is found in the microstrip lines layer. The mutual coupling between elements is more important in the slots and patches layer, while in the delay line layer (which is the layer which changes for each kind of lattice) the coupling is negligible.

As in the case of a single element, the results for a 2bit reflectarray element are shown, but now with gathering elements. This means that one half of the required MEMS and their associated DC network can be saved, with a significant reduction in the cost and the manufacturing complexity of large reflectarrays, and without any significant change in the performance of the element. Figure 10 shows the amplitude and phase of the reflection coefficient for the 2-bit element as a function of the frequency. The average losses of the four states for the phase-controllable gathered elements are $2.84 \mathrm{~dB}$, at the central frequency. As can be seen, the losses are not proportional to the number of switches because they also depend on the standing wave produced by the openended delay line and by the mismatch between the microstrip and the MEMS assembly. An almost linear phase response with the frequency can be observed at certain frequencies from the phase curves, giving rise to a True-Time Delay behaviour. Considering a phase error of $\pm 30^{\circ}$, which is a very restrictive value for a 2-bit phase-shifter, the bandwidth of the elements is $10 \%$ (from $9.50 \mathrm{GHz}$ to $10.60 \mathrm{GHz}$ ).

2.3. Wideband Reconfigurable Single Element Based on Two Stacked Patches, Aperture-Coupled to Delay Lines. A passive reflectarray element with more than $30 \%$ of bandwidth to be used in reconfigurable-beam antennas has been designed using the same configuration of aperture-coupled elements, but by adding an additional patch to enlarge the bandwidth 


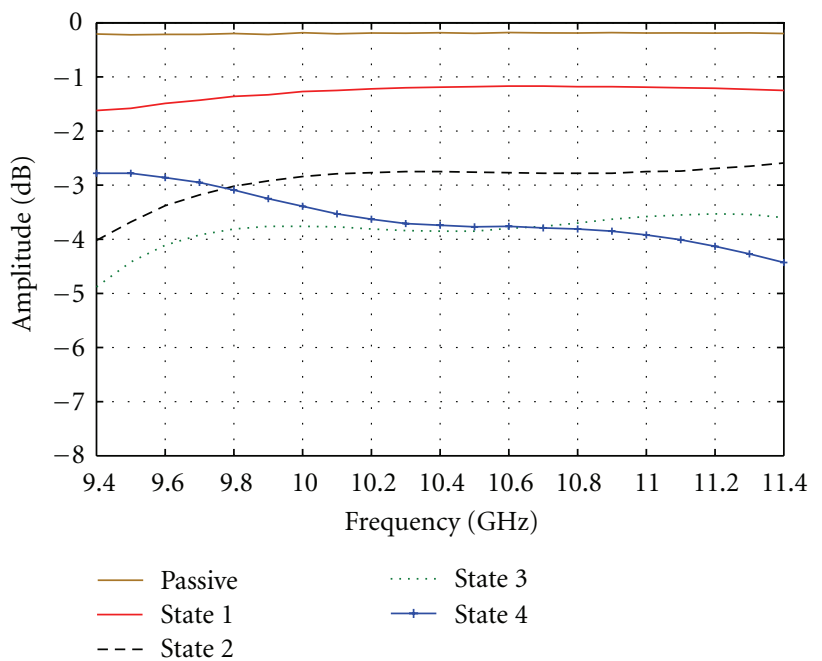

(a)

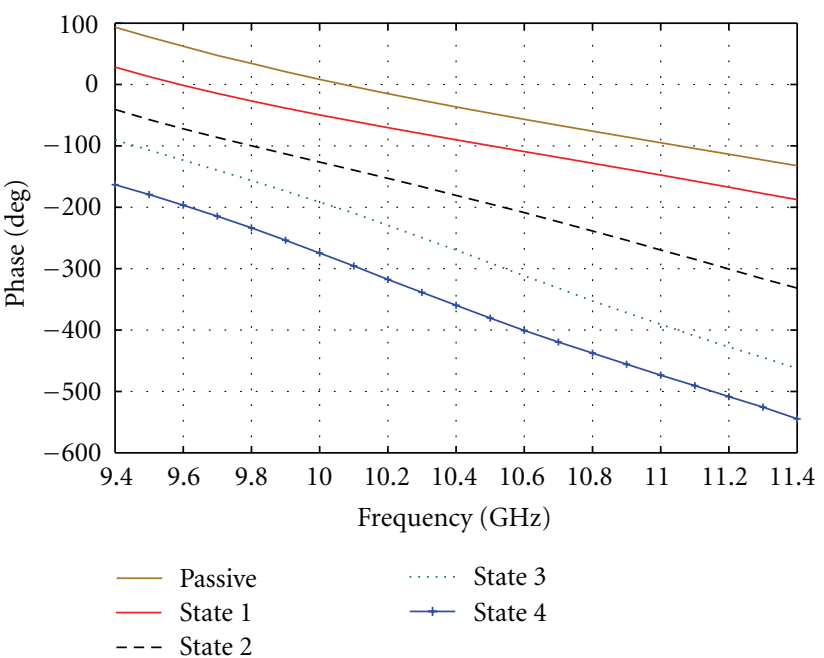

(b)

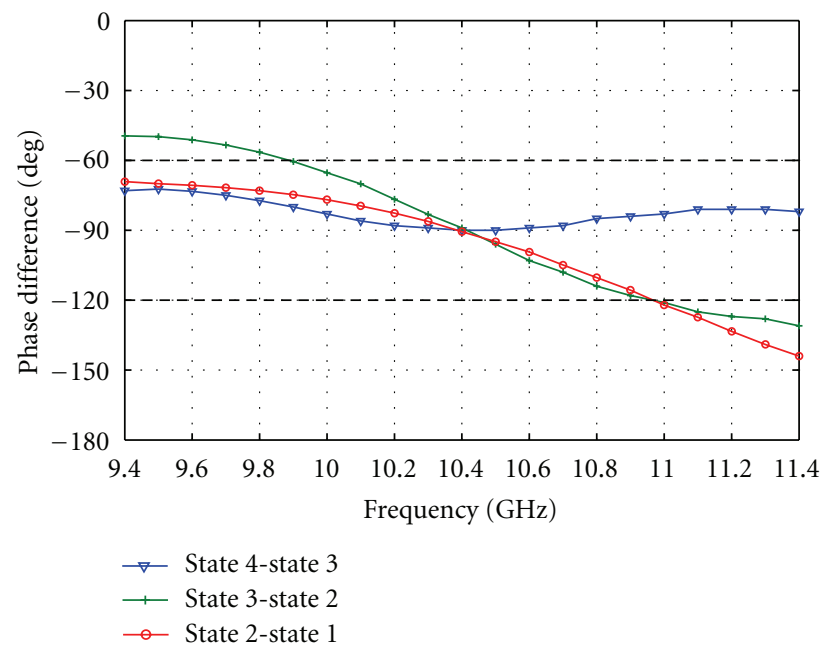

(c)

FIGURE 13: Reflection coefficient for the 2-bit wideband element with two stacked patches in a periodic environment. (a) Amplitude. (b) Phase. (c) Phase difference between the adjacent states.

as typically done in the case of elements printed arrays with stacked patches [28]. The proposed element is shown in Figure 11. As in previous cases, the element has been designed using a local periodicity approach with a period of $18.5 \mathrm{~mm} \times 18.5,(0.64 \lambda \times 0.64 \lambda)$, at $10.40 \mathrm{GHz}$. The square patches are $8.9 \times 8.9 \mathrm{~mm}^{2}$ and $6.23 \times 6.23 \mathrm{~mm}^{2}$ (upper patch $=0.7 \times$ lower patch), the slot $7.35 \times 1.5 \mathrm{~mm}^{2}$ and the delay line width $0.388 \mathrm{~mm}(70 \Omega)$. In this case, the matching stub has been fixed at $1.55 \mathrm{~mm}$, measured from the centre of the slot. The dielectric materials are the same as in Table 1. The additional materials have been defined as $\mathrm{d} 5=\mathrm{d} 3$ and $\mathrm{d} 6=\mathrm{d} 4$.

Figure 12 shows the reflection coefficient in free-space for the wideband element as a function of the delay line length for different frequencies. As can be seen, this element provides a practically linear phase delay in all of the analysed frequencies within a $30 \%$ bandwidth. If three MEMS are assembled along the delay line to forming a 2-bit phase shifter, the losses are much more stable in practically the entire band, compared with the original single-patch reflectarray element. The phase variations between adjacent states are also smaller than in the case of the single element with only one patch. In fact, the $\pm 30^{\circ}$ variation is fulfilled in practically the whole band. The amplitude, phase and phase variation between adjacent states can be seen in Figure 13. A tradeoff between the manufacturing difficulties generated by the need for the second stacked patch and the bandwidth requirements of each specific application must be made before deciding to use a single element with one patch or a single element with two stacked patches. It is clear that for the gathered element it is also possible to include an additional patch in order to increase the bandwidth. However it 
is also necessary to take into account the band limitations introduced by the T-junction and the impedance transformers which are needed in the case of a sub-array.

\section{Conclusions}

The feasibility of implementing surface-mounted MEMS switches in aperture-coupled reflectarray elements has been demonstrated in three cases: single elements, gathered elements, and a wideband single element with stacked patches. In all the cases, the effect of the MEMS assembly which includes wire bonding has been considered in the analysis.

The number of MEMS as well as their control network can be reduced through the grouping of adjacent reflectarray elements in pairs with a significant reduction in the cost and manufacturing complexity. The main drawback of the proposed gathering is the generation of grating lobes in the grouping direction, but its impact can be easily reduced by using irregular lattices.

Finally, the average losses of the elements can be drastically improved by reducing the series resistance of the MEMS switches to values of around $0.15 \Omega$ as well as reducing the mismatch between the microstrip lines and the bonding wires by manufacturing thinner MEMS devices. These are two technological issues on which the MEMS' manufacturer is working.

\section{Acknowledgments}

The work presented in this contribution has been supported in part by the Spanish Ministry of Economy and Competitiveness, under the Project Contracts TEC2010-17567, and by the Seventh Framework of the European Union, under the ARASCOM project FP7-ICT-2007-3.6:222620. The authors acknowledge CEA-LETI Minatec from France for providing the MEMS devices which were characterized and used in the simulations.

\section{References}

[1] M. Barba, E. Carrasco, J. E. Page, and J. A. Encinar, "Electronic controllable reflectarray elements in X band," Frequenz, vol. 61, no. 9-10, pp. 203-206, 2007.

[2] H. Kamoda, T. Iwasaki, J. Tsumochi, T. Kuki, and O. Hashimoto, "60-GHz electronically reconfigurable large reflectarray using single-bit phase shifters," IEEE Transactions on Antennas and Propagation, vol. 59, no. 7, pp. 2524-2531, 2011.

[3] E. Carrasco, M. Barba, and J. A. Encinar, "Demonstration of a switchable-beam reflectarray antenna with PIN diodes in X-band," in Proceedings of the 33rd ESA Antenna Workshop on Challenges for Space Antenna Systems (ESA/ESTEC '11), Noordwijk, The Netherlands, October 2011.

[4] B. Mencagli, R. V. Gatti, L. Marcaccioli, and R. Sorrentino, "Design of large mm-wave beam-scanning reflectarrays," in Proceedings of the 35th European Microwave Conference (EuMC '05), pp. 1875-1878, France, Paris, October 2005.

[5] H. Rajagopalan, Y. Rahmat-Samii, and W. A. Imbriale, "RF MEMS actuated reconfigurable reflectarray patch-slot element," IEEE Transactions on Antennas and Propagation, vol. 56, no. 12, pp. 3689-3699, 2008.
[6] J. Perruisseau-Carrier, F. Bongard, R. Golubovic-Niciforovic, R. Torres-Sanchez, and J. R. Mosig, "Contributions to the modeling and design of reconfigurable reflecting cells embedding discrete control elements," IEEE Transactions on Microwave Theory and Techniques, vol. 58, no. 6, pp. 1621-1628, 2010.

[7] O. Bayraktar, O. A. Civi, and T. Akin, "Beam switching reflectarray with MEMS controls," in Proceedings of the 4th European Conference on Antennas and Propagation (EuCAP '10), Barcelona, Spain, April 2010.

[8] E. Carrasco, M. Barba, B. Reig, C. Dieppedale, and J. A. Encinar, "Characterization of a reflectarray gathered element with electronic control using ohmic RF MEMS and patches aperture-coupled to a delay line," IEEE Transactions on Antennas and Propagation, vol. 60, no. 9, 2012.

[9] S. V. Hum, M. Okoniewski, and R. J. Davies, "Realizing an electronically tunable reflectarray using varactor diode-tuned elements," IEEE Microwave and Wireless Components Letters, vol. 15, no. 6, pp. 422-424, 2005.

[10] M. Riel and J. J. Laurin, "Design of an electronically beam scanning reflectarray using aperture-coupled elements," IEEE Transactions on Antennas and Propagation, vol. 55, no. 5, pp. 1260-1266, 2007.

[11] F. Venneri, S. Costanzo, and G. Di Massa, "Reconfigurable aperture-coupled reflectarray element tuned by single varactor diode," Electronics Letters, vol. 48, no. 2, pp. 68-69, 2012.

[12] R. R. Romanofsky, "Advances in scanning reflectarray antennas based on ferroelectric thin-film phase shifters for deepspace communications," Proceedings of the IEEE, vol. 95, no. 10, pp. 1968-1975, 2007.

[13] W. Hu, R. Cahill, J. A. Encinar et al., "Design and measurement of reconfigurable millimeter wave reflectarray cells with nematic liquid crystal," IEEE Transactions on Antennas and Propagation, vol. 56, no. 10, pp. 3112-3117, 2008.

[14] G. Pérez-Palomino, J. A. Encinar, M. Barba, and E. Carrasco, "Design and evaluation of multi-resonant unit-cells based on liquid crystals for reconfigurable reflectarrays," IET Microwaves, Antennas and Propagation, vol. 6, no. 3, pp. 348-354, 2012.

[15] E. Carrasco, M. Barba, and J. A. Encinar, "Reflectarray element based on aperture-coupled patches with slots and lines of variable length," IEEE Transactions on Antennas and Propagation, vol. 55, no. 3, pp. 820-825, 2007.

[16] E. Carrasco, J. A. Encinar, and M. Barba, "Bandwidth improvement in large reflectarrays by using true-time delay," IEEE Transactions on Antennas and Propagation, vol. 56, no. 8, pp. 2496-2503, 2008.

[17] J. Huang, "Bandwidth study of microstrip reflectarray and a novel phased reflectarray concept," in Proceedings of the IEEE Antennas and Propagation Society International Symposium, pp. 582-585, Newport Beach, Calif, USA, June 1995.

[18] E. Carrasco, M. Arrebola, J. A. Encinar, and M. Barba, "Demonstration of a shaped beam reflectarray using aperturecoupled delay lines for LMDS central station antenna," IEEE Transactions on Antennas and Propagation, vol. 56, no. 10, pp. 3103-3111, 2008.

[19] E. Carrasco, M. Barba, and J. A. Encinar, "Design and validation of gathered elements for steerable-beam reflectarrays based on patches aperture-coupled to delay lines," IEEE Transactions on Antennas and Propagation, vol. 59, no. 5, pp. 17561761, 2011.

[20] CEA LETI, http://www.leti.fr/.

[21] F. Souchon, P. L. Charvet, C. Maeder-Pachurka, and M. Audoin, "Dielectric charging sensitivity on MEMS switches," 
in Proceedings of the14th International Conference on SolidState Sensors, Actuators and Microsystems, pp. 363-366, Lyon, France, June 2007.

[22] N. Torres Matabosch, F. Coccetti, R. Plana, B. Reig, and J. L. Cazaux, "A High Isolation T-switch for reconfigurable switching matrix," in Proceedings of the 11th International Symposium on RF MEMS and RF Microsystems (MEMSWAVE '10), Otranto, ITALY, June 2010.

[23] CST Microwave Studio ${ }^{\circledR}$, http://www.cst.com/.

[24] D. Caratelli, M. C. Vigano, G. Toso, and P. Angeletti, "Analytical technique for constrained sparse array synthesis problems," in Proceedings of the 32nd ESA Antenna Workshop on Antennas for Space Applications, Noordwijk, The Netherlands, October 2010.

[25] H. Legay and B. Salome, "Low-loss reconfigurable reflector array antenna," U. S. Patent 7, 142, 164 B2, 2006.

[26] E. Carrasco, M. Barba, and J. A. Encinar, "Switchable-beam reflectarray antenna with gathered elements using different lattices," in Proceedings of the 6th Loughborough Antennas and Propagation Conference (LAPC '10), pp. 197-200, Loughborough, UK, November 2010.

[27] E. Carrasco, M. Arrebola, M. Barba, and J. A. Encinar, "Shaped-beam reconfigurable reflectarray with gathered elements in an irregular lattice for LMDS base station," in Proceedings of the 5th European Conference on Antennas and Propagation (EUCAP '11), pp. 975-978, Rome, Italy, April 2011.

[28] P. Gay-Balmaz, J. A. Encinar, and J. R. Mosig, "Analysis of multilayer printed arrays by a modular approach based on the generalized scattering matrix," IEEE Transactions on Antennas and Propagation, vol. 48, no. 1, pp. 26-34, 2000. 

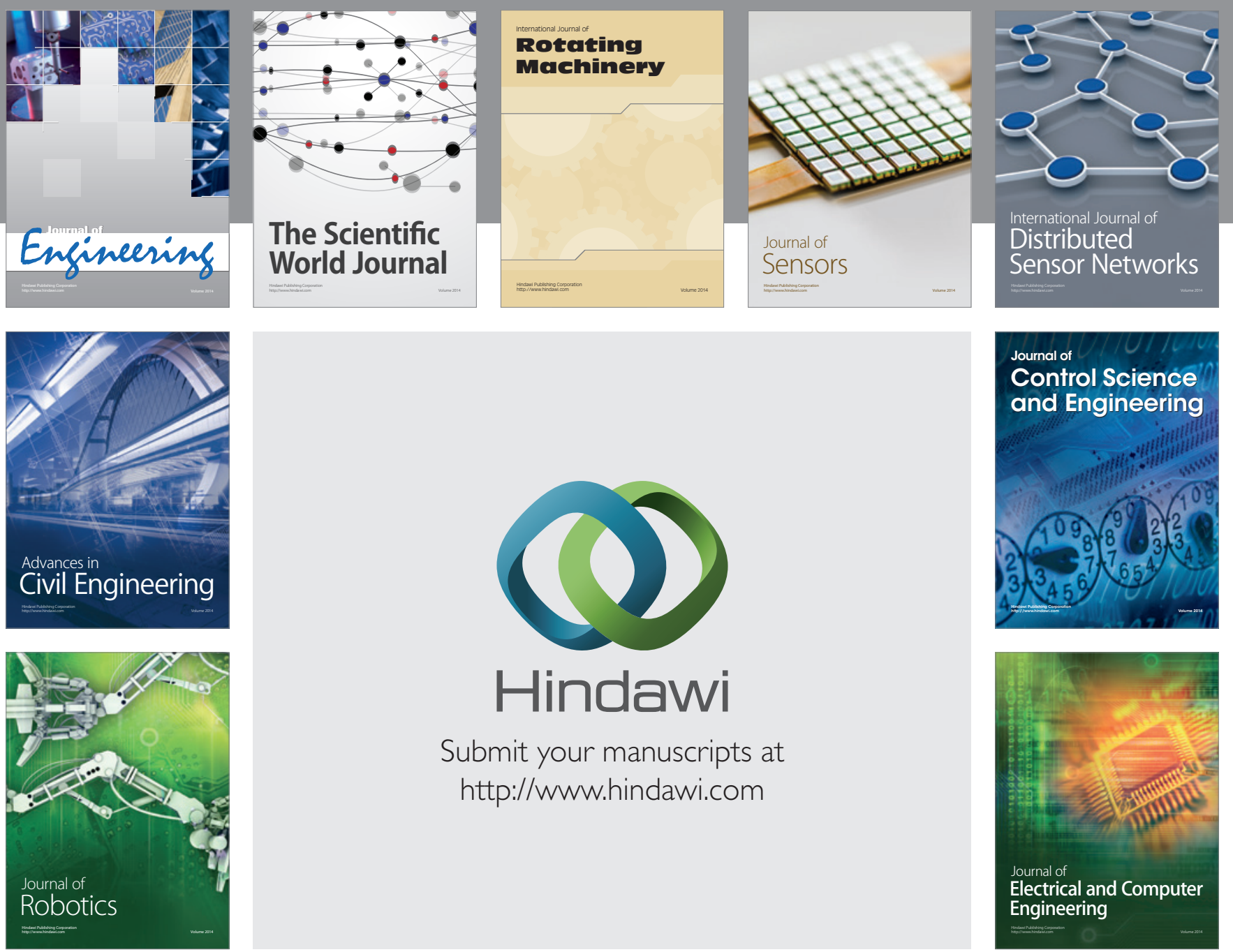

Submit your manuscripts at

http://www.hindawi.com
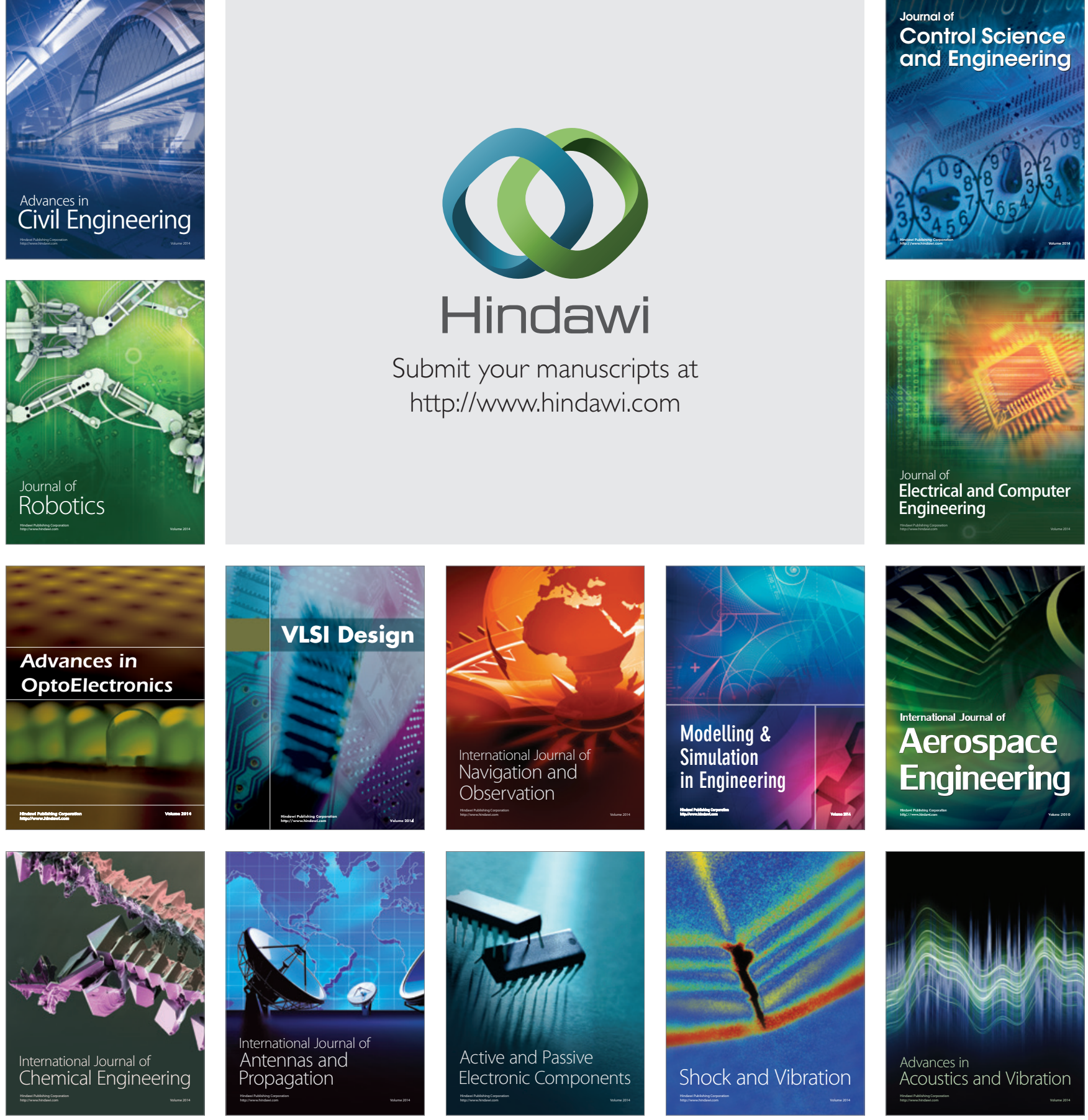